The recent IOM standards were developed by as best practice with little guidance for potential interpretation or resource requirements. The CMSS Principles are intended to be a step towards practical guidelines standards and this research the first feedback step as to measuring that practicality.

\section{P266 AT WHAT RATE DOES NEW EVIDENCE CHANGE GUIDANCE}

1J Ording, ${ }^{2} \mathrm{M}$ Thiese, ${ }^{2} \mathrm{~K}$ Hegmann, ${ }^{1} \mathrm{C}$ Wolfkiel, ${ }^{2} \mathrm{U}$ Ott. ${ }^{1}$ American College of Occupational and Environmental Medicine, Elk Grove Village, USA; ${ }^{2}$ University of Utah RMCOEH, Salt Lake City, USA

\section{0:1136/bmjqs-2013-002293.229}

Background New research is continually shaping guidelines; however, the rate of change has not been assessed.

Objectives Review articles from 2010 to 2013 to assess rate of change of guidelines for the elbow.

Methods A systematic literature search was conducted to identify randomised controlled trials (RCTs) on elbow disorders published between 2010 and 2013. Identified RCTs were scored using established scoring methods and incorporated into guidelines to determine if any recommendations needed to be changed or new recommendations added.

Results Fifteen new RCTs were identified (4 high-, 9 moderate-, and 2 low-quality). Nine (69\%) studies were used to make 3 new recommendations and 4 changes to recommendations to guidelines on elbow disorders. Seven of these studies prompted new guidance on soft tissue mobilisation, autologous blood injections, periarticular blood injections for lateral epicondylalgia (LE). Two of these studies caused changes to the recommendation level for manipulation/mobilisation for LE and evidence level changes for exercises, glucocorticosteroid injections, and platelet rich plasma injections for LE. Seven (53\%) studies did not change any of the recommendations but added to the body of evidence to support the current recommendations.

Discussion New studies may be higher quality and have significant impact on guidelines. Two-thirds of new evidence triggered recommendations changes or development of new recommendations for treating LE. Additional assessments of low back and other body parts are underway.

Implications for Guideline Developers/Users It is beneficial to do a yearly review of the literature to determine if any new evidence will impact changes to current guidelines.

\section{P268 WHAT KIND OF EVIDENCE SUPPORTED THE CLINICAL PRACTICE GUIDELINE FOR THE SYNDROMIC MANAGEMENT OF SEXUALLY TRANSMITTED INFECTIONS AND OTHER INFECTION OF THE GENITAL TRACT 2012}

1,2 H Gaitan, ${ }^{1,2} \mathrm{M}$ Torres, ${ }^{1} \mathrm{~A}$ Rodriguez. ${ }^{1}$ Clinical Research Institute, Universidad Nacional de Colombia, Bogota, Colombia; ${ }^{2}$ Sexually Transmitted Infections Cochrane Group, Bogota, Colombia

\section{0:1136/bmjqs-2013-002293.230}

Background In recent years, the GRADE approach has been broadly accepted by many GDG. There is a lack of information about the relationship between the types of evidence with the strength of recommendations using GRADE.
Objective To present the evidence mapping of the literature used to develop recommendations in the Guideline for Syndromic management of the Genital Tract Infections.

Methods The evidence of each recommendation was reviewed and was classified according to the type, quality and quantity of evidence and strength of the recommendation. A descriptive analysis was performed as well as a cross-analysis to find out the relationship between the strength of the recommendation and the underlying quality of the evidence.

Results 80 recommendations were identified. Systematic reviews supported the $29.1 \%$ of the recommendations, RCT $25.6 \%$, observational studies 5.9\%, guidelines $9.3 \%$ and expert opinion $30.1 \%$. The quality of the evidence was high (14\%), moderate $(15 \%)$, low (16\%) and very low (55\%). $63.7 \%$ of the recommendations were strong in favour. 14\% of the strong recommendations came from high quality evidence and 49\% came from very low quality evidence.

Discussion The evidence shows a similar percentage of systematic reviews, RCT and expert opinion in the guideline. Despite the quality of the evidence, the number of strong recommendations is high due to the other criteria of the GRADE approach. Implications for Guideline Developers The GRADE approach allows weighting other factors beyond the quality of the evidence. Research needs to be done on the most important factors in grading the recommendations.

\section{P269 ADAPTATION OF A NORTH AMERICAN INSTITUTIONALLY BASED HEALTH TECHNOLOGY ASSESSMENT (IHTA) MODEL TO A PRIVATE BRAZILIAN HEALTH CARE ORGANIZATION (BHCO)}

${ }^{1,2}$ S Alves Da Silva, ${ }^{1} \mathrm{M}$ Cabanelas Pazos, ${ }^{1} \mathrm{P}$ Pereira de Souza, ${ }^{1} \mathrm{P}$ Rascão Cardoso,

2,3,p Wyer. 'Amil Assistência Médica Internacional, Rio de Janeiro, Brazil; ${ }^{1}$ The New York Academy of Medicine, New York, USA; ${ }^{3}$ Columbia University, New York, USA

\section{0:1136/bmjqs-2013-002293.231}

Background Brazilian Guidelines, developed by medical societies, are sparsely used by federal agencies to determine coverage. To date there is no organised approach to clinical policy and guideline development or for dialogue with regulators within BHCOs. Amil, the largest BHCO, covers 6 million lives distributed across 8 regions and delivers care to many through its own medical centres.

Objectives To develop a minimally resourced clinical policy and implementation capability within Amil together with a training programme on a national level.

Methods Our approach is based on observation of the Penn Health System and the Kaiser Permanente (KP) models of IHTA. We are profiling current capacity for integrating umbrella reviews with mining and interpretation of internally generated practice data, and are identifying resource and manpower needs. To promote cultural change on national scale we reformulated an annual training workshop made in partnership with NYAM and McMaster and opened to participants within and without Amil, including Health Ministry and Regulatory Agencies, by addressing guideline development, adaptation and implementation skills.

Results Our approach identifies knowledge gaps within the organisation and develops related guidelines and outcomes assessment to be internally used through Electronic Health Records and to be presented to regulators as proposal for change. The framework was built on a piloted approach on 